Research Article

\title{
Determination of Optimal Warranty Period with Preventive Maintenance Actions for Items from Heterogeneous Populations
}

\author{
Minjae Park (i) \\ College of Business Administration, Hongik University, Seoul, Republic of Korea \\ Correspondence should be addressed to Minjae Park; mjpark@hongik.ac.kr
}

Received 27 July 2020; Revised 18 October 2020; Accepted 28 October 2020; Published 11 November 2020

Academic Editor: Arturo J. Fern ndez

Copyright (c 2020 Minjae Park. This is an open access article distributed under the Creative Commons Attribution License, which permits unrestricted use, distribution, and reproduction in any medium, provided the original work is properly cited.

\begin{abstract}
In this study, we develop an optimal maintenance policy with replacement service and minimal repair service for items from heterogeneous populations and determine the optimal warranty length and repair time threshold. We consider the informationbased repair-replacement policy model and develop the formula to evaluate the expected cost rate during the product life cycle. A general formulation is derived for the expected cost rate under a warranty policy for items of heterogeneous populations. When a replacement service and minimal repair service are provided for a failed item, then an item from a weak population has the property of an item from a weak population after service. Similarly, an item from a strong population has the property of an item from a strong population after service. We define the optimal maintenance strategies to minimize the expected cost rate with failure time and repair time for items with heterogeneous reliability characteristics. The effects of parameters of the intensity function for the failure times on the optimal length of the warranty period are studied numerically. Assuming that the product deteriorates, we illustrate the proposed approach using numerical applications and observe the impacts of relevant parameters on the optimal length of the warranty period.
\end{abstract}

\section{Introduction}

The research for the maintenance cost under warranty has been conducted and maintenance models under two-dimensional warranty have been developed intensively in the literature (see, e.g., [1-15]). Recently, many researchers have developed two-dimensional warranty with preventive maintenance (PM) actions considering different factors like minimal repair $[1,4,6-10]$, age and usage $[4,6,7]$, repairable item/product $[1,2,4,7,8,11]$, manufacturer's perspective $[1,4,5,16]$, customer's perspective $[6,12-14]$, extended warranty $[1-4,6,14]$, profit maximization $[6,7]$, prorata warranty $[2,9,13]$, free-repair warranty $[9,11,13]$, postwarranty period $[9,10,12-15]$, renewable warranty $[10,12]$, nonrenewable warranty $[13,14]$, periodic PM $[2,5,7,9,12]$, nonperiodic PM $[2,8,9]$, and imperfect PM $[1,7]$. Different features for the two-dimensional warranty can be found in Park and Pham $[9,13]$ and Park et al. [16-19] in which failure times and repair times are considered as two dimensions for warranty policy. PM effects can be implemented in different ways using virtual age $[4,15]$, age reduction, and others. Wang and Su [5] suggested a new PM policy called two dimensional PM strategy in the two dimensional warranty. Jung et al. [14] developed a two-phase warranty and determined the optimal length of the maintenance period by minimizing the expected total cost after the extended warranty expires. Wang et al. [1] developed an optimal PM policy with the base and extended warranties from the manufacturer's perspective. Park et al. [10] proposed a renewing free repair-replacement warranty (FRRW) to derive an optimal maintenance strategy for a repairable product after the warranty expires. Under the FRRW, failure time and repair time are considered together to carry out the warranty service for failed products by setting a time limit of warranty service during the warranty period.

To reduce the risk of product failures, various types of maintenance actions are carried out, particularly for repairable products. These maintenance actions may generally be classified into three types: PM, corrective maintenance $(\mathrm{CM})$, and condition-based maintenance $(\mathrm{CBM})$. For a 
deteriorating but repairable system, $\mathrm{PM}$ actions are performed at planned times to improve the reliability of the item, whereas CM is conducted upon failure to recover the system to working status and CBM is provided depending on the product's condition. Most research on maintenance policies with both a PM action and a CM action has been carried out under the assumption that an item's population is homogeneous with respect to reliability characteristics, whereby the lifetime of the items can be described by the same probability distribution. However, populations are usually heterogeneous. The heterogeneous population has been critical in academic and practical fields such as reliability modelling. Implementing heterogeneity into the framework of cost modelling is challenging. Since Finkelstein [20] developed the model with minimal repairs in heterogeneous population, many studies [21-24] on the statistical properties of heterogeneous populations have been carried out. Cha and Finkelstein $[22,24]$ assumed that the items' population consists of two subpopulations and Cha and Finkelstein [22] also analysed the effect of PM on the reliability features of items selected from heterogeneous populations. Lee et al. [23] and Cha [21] developed a warranty policy for items which come from a heterogeneous population. In their model, an item is randomly selected from a heterogeneous population which consists of weak subpopulation and strong subpopulation.

Recently, Yang et al. [25] investigate a novel two-phase preventive maintenance policy for a single-component system with an objective of maximizing the revenue generated by the performance-based contracting. Peng et al. [26] study a single-unit system subject to two types of failures whereas preventive replacements are implemented to avoid any potential failure due to the catastrophic failure mode.

In this paper, we deal with the warranty policy for PM strategy for heterogeneous items from the manufacturer's perspective. We develop a cost model considering repair and replacement as a warranty service. If a repair service time exceeds the prefixed repair time limit, then a replacement service for failed items is provided, not continuing to provide a repair service. It indicates that a user does not need to wait for a long time and it is possible for dealers to deal with costs and customer dissatisfaction caused when repairing an unfixed product continuously. However, because a replacement cost would be higher than a repair cost generally, dealers have to make a good strategy.

If the maintenance cost is included in the warranty cost, then the warranty costs can be reduced by conducting effective PM actions during the warranty period. The reliability improvement of an item by a PM action could reduce the warranty costs, although a PM action can impose additional costs. One of the effective ways to reduce warranty cost is to conduct appropriate maintenance actions for the product's quality and reliability during the warranty period [4].

A minimal repair indicates that the state of the product after the repair is restored to the state prior to the failure. After a minimal repair action, the product is working but the failure rate of the product remains unchanged.
Consequently, the nonhomogeneous Poisson process (NHPP) is usually used to model the failure process when minimal repair is considered [11]. It is more reasonable to consider that item comes from heterogeneous populations, not homogeneous populations. Different kinds of the modelling framework [4] are implemented to serve the research purpose in this study. It is meaningful to consider repair and replacement together and items' heterogeneous population, so we develop the maintenance model for items from a heterogeneous population under the FRRW.

The settings and contributions of this paper differ from the existing literature in the following aspects: (1) a twodimensional warranty for the item from heterogeneous populations with repair time and failure time is proposed; (2) item under warranty from heterogeneous populations with warranty services such as minimal repair service and replacement service is considered; (3) PM strategy for the item under warranty from heterogeneous populations is developed and its statistical properties are investigated. In this paper, we develop the repair-replacement policy with a repair time threshold for an item from a heterogeneous population to consider the decision variable and to minimize total cost. We determine the optimal length of the warranty period for items from a heterogeneous population with minimal repair.

The remainder of this paper is organized as follows. Section 2 explains the problem in detail. Section 3 describes the repair-replacement policy and gives detailed discussions on the cost models considered in this paper. Section 3 shows the mathematical formulas derived to evaluate the expected cost rate (ECR). In Section 4, we illustrate the proposed models and optimal solutions by numerical examples, considering the power law process for the product failures and a Weibull distribution for the repair times, respectively. The sensitivity analysis for the optimal length of the warranty period is also given numerically in Section 4. Finally, concluding remarks are given in Section 5.

\section{Problem Description}

\subsection{Assumptions}

(1) The minimal repair does not modify the failure rate of an item; however, we may observe failure information, defining the state of an item. This may result in a more general type of repair, which is called the information-based minimal repair [20, 24, 27, 28]. If it was an item from the strong (weak) subpopulation before the failure, then after a minimal repair, it also remains strong (weak) [23]. This kind of minimal repair can be considered as the information-based minimal repair. Information-based minimal repair is considered in this study.

(2) An item is defined by something that is part of a product or things. A new item is randomly selected from the heterogeneous population and the population from which the item is selected is not known.

(3) For the failed item, minimal repair service is provided under the nonrenewing warranty with the 
warranty period A and PM actions are scheduled at a fixed time $\delta<\omega$ without any cost to the customer in the warranty period.

(4) After PM actions, the failure rate for the item would be decreased due to PM effects.

(5) Whenever a failure occurs for an item, then minimal repair service is provided first. Repair time exceeds the repair time threshold; then, replacement service is provided, not continuing to provide the repair service.

2.2. Heterogeneous Population. The subpopulation of items is termed the "weak subpopulation," as its lifetime is shorter than other subpopulations stochastically. We denote its cumulative distribution function $(c d f)$, probability density function $(p d f)$, survival function, and failure rate function as $f_{1}(\cdot), F_{1}(\cdot), \bar{F}_{1}(\cdot)$, and $\lambda_{1}(\cdot)$, respectively. On the other hand, if its lifetime is longer than that of other subpopulations then it will be called the strong subpopulation. Similarly, its $c d f, p d f$, survival function, and failure rate function are $f_{2}(\cdot), F_{2}(\cdot), \bar{F}_{2}(\cdot)$, and $\lambda_{2}(\cdot)$, respectively. The proportion of the weak items is $\pi_{1}$, whereas the proportion of the strong items is $\pi_{2}=1-\pi_{1}$, and the mixture survival function denoted by $\bar{F}(t)$ is given as $\bar{F}(t)=\pi_{1} \bar{F}_{1}(t)+\pi_{2} \bar{F}_{2}(t)$. A new item is randomly chosen from the heterogeneous population and it is composed of strong subpopulation and weak subpopulation. It is considered that the subpopulation from which the item was selected is unknown. An item is preventively maintained at a fixed time of $\delta<\omega$ without any cost to the buyers in $[0, \omega]$. Thus, we consider a free repair-replacement policy. Through this study, the failure rates corresponding to each subpopulation increase significantly and $\lambda_{1}(t)>\lambda_{2}(t)$.

2.3. Repair-Replacement Policy for Heterogeneous Items. In this study, we determine the optimal warranty length for items from a heterogeneous population. Item can be defined by one component or one part and if a product can be provided after assembling components or parts which are items. We develop the model for an item, and usually, an item is assumed to have the same reliability but, in real life, it has a different reliability in terms of the life cycle. After the minimal repair service is provided for the failed item, then an item from the strong population has the same property as an item from the strong population after minimal repair service. Similarly, an item from the weak population has the same property as an item from the weak population after minimal repair.
We consider a repair-replacement policy under warranty for items from a heterogeneous population. Lee et al. [23] and Cha [21] assumed that the item is randomly selected from a heterogeneous population which is composed of strong subpopulation and weak subpopulation. The periodic heterogeneous replacement for deteriorating items is used from a heterogeneous population.

Under the information-based repair-replacement policy $[20,24,27,28]$, a newly installed product is maintained preventively at the predetermined cycle $\delta$ subject to no failures occurring during the warranty period. However, if the product failures occur during the warranty period, then the product is minimally repaired first. If a repair service can be completed within the repair time threshold $r_{0}$, then the product is restored to "bad-as-old." Otherwise, the failed item is replaced with a brand-new item. Consider a certain situation for which a customer purchases a high-priced product, whereby if a product failure occurs, the customer suffers a substantial monetary loss. In this situation, a customer wishes to keep the product operating without failure during the mission period. However, the customer would prefer minimal repair to immediate replacement on the product failure if the product can be repaired within a reasonably short period of time. It is generally true that replacement cost is considerably higher than the minimal repair cost. The repair-replacement policy with the repair time threshold has been discussed in Park et al. [10] in more general situations.

\section{Model Analysis}

3.1. Information-Based Minimal Repair for Items from Heterogeneous Population. The information-based repair-replacement policy could act as a more profitable maintenance policy than the basic repair-replacement policy under situations. In actual applications, this type of informationbased minimal repair is more realistic [29]; therefore, hereafter, we assume this information-based minimal repair for the items from the heterogeneous population. Using the information-based minimal repair, we develop a repairreplacement policy to determine a decision variable to minimize the expected cost rate. Let $\delta$ and $\omega$ denote the PM cycle and length of the warranty period, respectively, and let $L(\omega)$ represent the elapsed time to the first replacement of the item from a heterogeneous population. Note that the process of minimal repairs for items follows the NHPP. With consideration of the repair time threshold $r_{0}, L(\omega)$ can be expressed as [16]

$$
L(\omega)= \begin{cases}T, & T<\omega, Y>r_{0}, \\ \omega, & T \geq \omega \text { or all failures before } \omega \text { are information - based minimally repaired } .\end{cases}
$$


We must find the reliability function of $L(\omega)$ in order to obtain the expectation for $L(\omega)$. Then, the reliability function of $L(\omega)$ can be written as [30]

$$
\begin{aligned}
P(L(\omega)>t) & =\frac{\mathrm{H}(0, t)^{0} e^{-\mathrm{H}(0, t)}}{0 !}+\frac{\mathrm{H}(0, t)^{1} e^{-\mathrm{H}(0, t)}}{1 !} G\left(r_{0}\right)+\frac{\mathrm{H}(0, t)^{2} e^{-\mathrm{H}(0, t)}}{2 !}\left[G\left(r_{0}\right)\right]^{2}+\cdots \\
& =e^{-\mathrm{H}(0, t)} \sum_{k=0}^{\infty} \frac{\left[\mathrm{H}(0, t) G\left(r_{0}\right)\right]^{k}}{k !} \\
& =[\bar{F}(t)]^{\bar{G}\left(r_{0}\right)} .
\end{aligned}
$$

The last equality follows from the fact that $e^{-\mathrm{H}(0, t)}=e^{-\int_{0}^{t} h(u) \mathrm{d} u}=\bar{F}(t)$. If we use the heterogeneous survival function from heterogeneous populations, then the survival function of $L(\omega)$ becomes $P(L(\omega)>t)=\left(\pi_{1} \bar{F}_{1}(t)+\pi_{2} \bar{F}_{2}(t)\right)^{\bar{G}\left(r_{0}\right)}$. It follows that the expected time to the first replacement of the product can be given by

$$
E[L(\omega)]=\int_{0}^{\omega}\left(\pi_{1} \bar{F}_{1}(t)+\pi_{2} \bar{F}_{2}(t)\right)^{\bar{G}\left(r_{0}\right)} \mathrm{d} t .
$$

When $r_{0}$ equals 0 and $\bar{G}(0)=1, E[L(\omega)]$ is thus reduced to

$$
E[L(\omega)]=\int_{0}^{\omega}\left(\pi_{1} \bar{F}_{1}(t)+\pi_{2} \bar{F}_{2}(t)\right) \mathrm{d} t
$$

We then derive the expected cost rate during the warranty period. Let $N(a, b)$ denote the number of failures of an item in the interval $(a, b)$. When $c_{m}$ and $c_{r}$ stand for unit minimal repair and replacement cost, respectively, then, given $L(\omega)=x$, the total cost incurred during the life cycle of the product, denoted by $C(\omega)$, can be expressed as

$$
C(\omega)= \begin{cases}c_{m} \cdot N(0, x)+c_{r}, & x<\omega, \\ c_{m} \cdot N(0, \omega), & x \geq \omega .\end{cases}
$$

To evaluate the expected number of minimal repairs, we let $\Lambda(a, b)=\int_{a}^{b} \lambda(u) \mathrm{d} u$ be the expected number of failures during the interval $[a, b]$ before the replacement takes place. Since all the failures are only minimally repaired prior to the replacement, no repair time exceeds the repair time threshold $r_{0}$. Thus, we obtain

$$
\begin{aligned}
& E[N(a, b)]=\sum_{k=1}^{\infty} k \cdot P(N(a, b)=k) \\
& =\sum_{k=1}^{\infty} k \cdot \frac{\Lambda(a, b)^{k} e^{-\Lambda_{m}(a, b)}}{k !}\left[G\left(r_{0}\right)\right]^{k} \\
& =\Lambda(a, b) \cdot G\left(r_{0}\right) \cdot\left[e^{-\Lambda(a, b)}\right]^{\bar{G}\left(r_{0}\right)} .
\end{aligned}
$$

If there are no PM actions during the warranty period, then the expected number of minimal repairs can be given by

$$
E[N(0, \omega)]=\Lambda(0, \omega) \cdot G\left(r_{0}\right) \cdot\left[e^{-\Lambda(0, \omega)}\right]^{\bar{G}\left(r_{0}\right)}
$$

where $\Lambda(0, \omega)=\int_{0}^{\omega} \lambda(u) \mathrm{d} u$.

If there is no PM action available, then the expected minimal repair cost, $\operatorname{EMC}(\omega)$, can be given by

$$
\operatorname{EMC}(\omega)=c_{m} \int_{0}^{\omega} \lambda(u) \mathrm{d} u \cdot G\left(r_{0}\right) \cdot\left[e^{-\Lambda(0, \omega)}\right]^{\bar{G}\left(r_{0}\right)} .
$$

If there is no PM action available, then the expected warranty cost, $\mathrm{EC}(\omega)$, based on equation (5) can be given by

$$
\begin{aligned}
\operatorname{EC}(\omega)= & \left(c_{m} \int_{0}^{x} \lambda(u) \mathrm{d} u \cdot G\left(r_{0}\right) \cdot\left[e^{-\Lambda(0, x)}\right]^{\bar{G}\left(r_{0}\right)}+c_{r}\right) \\
& \cdot F(\omega)+c_{m} \int_{0}^{\omega} \lambda(u) \mathrm{d} u \cdot G\left(r_{0}\right) \cdot\left[e^{-\Lambda(0, \omega)}\right]^{\bar{G}\left(r_{0}\right)} \cdot \bar{F}(\omega) .
\end{aligned}
$$

For the heterogeneous setting, under the repair-replacement warranty, from the characteristics of minimal repair, the conditional expectation of the number of failure 
for an item from a weak subpopulation during the interval $[0, \omega]$ can be given by

$$
\begin{aligned}
E\left[N(0, \omega) \mid \pi=\pi_{1}\right] & =\int_{0}^{\omega} \lambda_{1}(u) \mathrm{d} u \cdot G\left(r_{0}\right) \cdot\left[e^{-\Lambda_{1}(0, \omega)}\right]^{\bar{G}\left(r_{0}\right)} \\
& =\Lambda_{1}(0, \omega) \cdot G\left(r_{0}\right) \cdot\left[e^{-\Lambda_{1}(0, \omega)}\right]^{\bar{G}\left(r_{0}\right)},
\end{aligned}
$$

where

$$
\Lambda_{1}(0, \omega)=\int_{0}^{\omega} \lambda_{1}(u) \mathrm{d} u .
$$

Similarly, the number of failures for an item from a strong subpopulation during the interval $[0, \omega]$ can be given by

$$
\begin{aligned}
E\left[N(0, \omega) \mid \pi=\pi_{2}\right] & =\int_{0}^{\omega} \lambda_{2}(u) \mathrm{d} u \cdot G\left(r_{0}\right) \cdot\left[e^{-\Lambda_{2}(0, \omega)}\right]^{\bar{G}\left(r_{0}\right)} \\
& =\Lambda_{2}(0, \omega) \cdot G\left(r_{0}\right) \cdot\left[e^{-\Lambda_{2}(0, \omega)}\right]^{\bar{G}\left(r_{0}\right)},
\end{aligned}
$$

where

$$
\Lambda_{2}(0, \omega)=\int_{0}^{\omega} \lambda_{2}(u) \mathrm{d} u .
$$

Let $\lambda_{i}(t)$ denote failure rate function for an item from subpopulation $i$ ( $i=1$, weak population, and $i=2$, strong population) and $\lambda_{i}^{p}(t)$ failure rate function after a PM action is conducted and $\Lambda_{i}^{p}(a, b)=\int_{b}^{a} \lambda_{i}^{p}(t) \mathrm{d} t$. When only one PM action is considered with PM cycle $\delta$, the expected warranty cost during the warranty period can be obtained by

$$
\begin{aligned}
\operatorname{EC}(\omega)= & \pi_{1} c_{m}\left[\int_{0}^{\delta} \lambda_{1}(u) \mathrm{d} u \cdot G\left(r_{0}\right)\left[e^{-\Lambda_{1}(0, \delta)}\right]^{\bar{G}\left(r_{0}\right)}+\int_{\delta}^{\omega} \lambda_{1}^{p}(u) \mathrm{d} u \cdot G\left(r_{0}\right)\left[e^{-\Lambda_{1}^{p}(\delta, \omega)}\right]^{\bar{G}\left(r_{0}\right)}\right] \\
& +\pi_{2} c_{m}\left[\int_{0}^{\delta} \lambda_{2}(u) \mathrm{d} u \cdot G\left(r_{0}\right)\left[e^{-\Lambda_{2}(0, \delta)}\right]^{\bar{G}\left(r_{0}\right)}+\int_{\delta}^{\omega} \lambda_{2}^{p}(u) \mathrm{d} u \cdot G\left(r_{0}\right)\left[e^{-\Lambda_{2}^{p}(\delta, \omega)}\right]^{\bar{G}\left(r_{0}\right)}\right]+c_{\mathrm{pm}} .
\end{aligned}
$$

We develop a repair-replacement policy using information-based minimal repair service for items from a heterogeneous population and investigate the properties for the policy. The repair-replacement policy with the information-based minimal repair is completely characterized by a set of three parameters $(\gamma, \delta, \omega)$ where $\gamma$ stands for the classification threshold level for strong and weak subpopulations. We develop the expected warranty cost function denoted by $C(\omega)$ and consider obtaining the optimal set of parameters to minimize the expected warranty cost for the item from a heterogeneous population. Along with the goal of optimizing the PM time and the length of the warranty period which are the main parameters for optimization of the model, we optimize the classification threshold $\gamma . s_{j}, j=$ $1,2, \ldots$, denotes $j^{\text {th }}$ failure time in $\left(0, L(\omega)\right.$.] Let $H_{[0, \omega]}$ be the history in $[0, \omega]$ of the operating and minimally repaired item and $h_{[0, \omega]}$ be the corresponding realization. Then, given $H_{[0, \omega]}=h_{[0, \omega]}$, the PM policy under warranty can be defined as follows: (a) If $h_{[0, \omega]} \in \mathrm{H}_{\gamma}$, the item operates further and whenever a failed item occurs in the interval $[0, \omega]$, then the information-based minimal repair service is provided until its repair time limit.

(b) If a repair service is not completed up to the repair time limit, then a replacement service is provided, not continuing to provide a repair service.

(c) Otherwise, the item is preventively maintained at the time $n \delta, n=0,1,2, \ldots$, during the warranty period.

If the probability that the item was chosen from the strong subpopulation is high, which is equal to be larger than the threshold level $\gamma$, the PM action for the items from the strong subpopulation is provided. If the probability that the item was chosen from the strong subpopulation is low, which is equal to be smaller than the threshold level $\gamma$, the PM action for the item from the weak subpopulation is provided. The joint distribution of failure during the life cycle of a product for subpopulation $i(i=1$, weak population and $i=2$, strong population, resp.) is given by

$$
\begin{aligned}
& \prod_{j=1}^{n} \lambda_{i}\left(s_{j}\right) \cdot \exp \left\{-\Lambda_{i}(0, L(\omega))\right\} \\
& =\lambda_{i}\left(s_{1}\right) \exp \left(-\Lambda_{i}\left(0, s_{1}\right)\right) \ldots \lambda_{i}\left(s_{n}\right) \exp \left\{-\Lambda_{i}\left(s_{n-1}, s_{n}\right)\right\} \exp \left\{-\Lambda_{i}\left(s_{n}, L(\omega)\right)\right\} .
\end{aligned}
$$


The probability that the randomly selected item is from a subpopulation, $i=1,2$, given that $L(\omega)$ is larger than or equal to the warranty period, is given by

$$
P\left(\pi=\pi_{i}, L(\omega) \geq \omega \mid H_{[0, \omega]}=h_{[0, \omega]}\right)=\frac{\pi_{i} \prod_{j=1}^{n} \lambda_{i}\left(s_{j}\right) \exp \left\{-\Lambda_{i}(0, \omega)\right\}}{\pi_{1} \prod_{j=1}^{n} \lambda_{1}\left(s_{j}\right) \exp \left\{-\Lambda_{1}(0, \omega)\right\}+\pi_{2} \prod_{j=1}^{n} \lambda_{2}\left(s_{j}\right) \exp \left\{-\Lambda_{2}(0, \omega)\right\}} .
$$

The probability that the randomly selected item is from a subpopulation, $i=1,2$, given that $L(\omega)$ is smaller than the warranty period, is given by

$$
P\left(\pi=\pi_{i}, L(\omega)<\omega \mid H_{[0, \omega]}=h_{[0, \omega]}\right)=\frac{\pi_{i} \prod_{j=1}^{n} \lambda_{i}\left(s_{j}\right) \exp \left\{-\Lambda_{i}(0, L(\omega))\right\}}{\pi_{1} \prod_{j=1}^{n} \lambda_{1}\left(s_{j}\right) \exp \left\{-\Lambda_{1}(0, L(\omega))\right\}+\pi_{2} \prod_{j=1}^{n} \lambda_{2}\left(s_{j}\right) \exp \left\{-\Lambda_{2}(0, L(\omega))\right\}}
$$

Therefore, the probability in which the randomly selected item coming from the weak subpopulation is smaller than the threshold $\gamma$ is given by

$$
\begin{aligned}
& P\left(\pi=\pi_{1}, L(\omega) \geq \omega \mid H_{[0, \omega]}=h_{[0, \omega]}\right) \\
& +P\left(\pi=\pi_{1}, L(\omega)<\omega \mid H_{[0, \omega]}=h_{[0, \omega]}\right)<\gamma,
\end{aligned}
$$

which can be equivalently expressed as

$$
\frac{\bar{F}_{1}\left(\pi_{1}\right) \cdot \pi_{1}}{\bar{F}_{1}\left(\pi_{1}\right) \cdot \pi_{1}+\left[\prod_{j=1}^{n} \lambda_{2}\left(s_{j}\right) / \lambda_{1}\left(s_{j}\right)\right] \cdot \bar{F}_{2}\left(\pi_{2}\right) \cdot \pi_{2}}<\gamma,
$$

which now characterizes set $H_{n}, n \geq 1$. In a similar way, for $n=0$, taking into account the convention, we have

$$
\frac{\bar{F}_{1}\left(\pi_{1}\right) \cdot \pi_{1}}{\bar{F}_{1}\left(\pi_{1}\right) \cdot \pi_{1}+\bar{F}_{2}\left(\pi_{2}\right) \cdot \pi_{2}}<\gamma .
$$

Therefore, the probability in which the randomly selected item coming from the strong subpopulation is larger than and equal to the threshold $\gamma$ is given by

$$
P\left(\pi=\pi_{2}, L(\omega) \geq \omega \mid H_{[0, \omega]}=h_{[0, \omega]}\right)+P\left(\pi=\pi_{2}, L(\omega)<\omega \mid H_{[0, \omega]}=h_{[0, \omega]}\right) \geq \gamma
$$

Which can be equivalently expressed as

$$
\frac{\bar{F}_{2}\left(\pi_{2}\right) \cdot \pi_{2}}{\left[\prod_{j=1}^{n} \lambda_{1}\left(s_{j}\right) / \lambda_{2}\left(s_{j}\right)\right] \cdot \bar{F}_{1}\left(\pi_{1}\right) \pi_{1}+\bar{F}_{2}\left(\pi_{2}\right) \cdot \pi_{2}} \geq \gamma,
$$

and it now characterizes set $H_{n}, n \geq 1$. In a similar way, for $n=0$, taking into account the convention, we have

$$
\frac{\bar{F}_{2}\left(\pi_{2}\right) \cdot \pi_{2}}{\bar{F}_{1}\left(\pi_{1}\right) \pi_{1}+\bar{F}_{2}\left(\pi_{2}\right) \cdot \pi_{2}} \geq \gamma .
$$

Let $T$ denote the failure time of the product with the PM action during the warranty period. Then, $p d f$ and $c d f$ of $T$ can be expressed as functions of $\lambda(t)$ as follows:

$$
\begin{aligned}
& f(t)=\lambda(t) \cdot \quad \exp \{-\Lambda(0, t)\}, \\
& F(t)=1-\exp \{-\Lambda(0, t)\} .
\end{aligned}
$$

Suppose that a failure rate function $\lambda(t)$ increases and the PM interval is assumed to be the same. If one PM action is considered only, we derive that the expected minimal repair cost during the warranty period is given by

$$
C_{m}(\omega)= \begin{cases}c_{m}\left\{(\Lambda(0, \delta)+\Lambda(\delta, L(\omega))) G\left(r_{0}\right) \bar{F}(L(\omega))^{\bar{G}\left(r_{0}\right)}+\Lambda(0, L(\omega)) G\left(r_{0}\right)[\bar{F}(L(\omega))]^{\left.\bar{G}\left(r_{0}\right)\right\},}\right. & L(\omega) \leq \omega, \\ c_{m}\left\{(\Lambda(0, \delta)+\Lambda(\delta, \omega)) G\left(r_{0}\right) \bar{F}(\omega)^{\bar{G}\left(r_{0}\right)}+\Lambda(0, \omega) G\left(r_{0}\right)[\bar{F}(\omega)]^{\left.\bar{G}\left(r_{0}\right)\right\},}\right. & L(\omega)>\omega .\end{cases}
$$


Let $\operatorname{EN}_{i}(a, b)$ denote the expected number of failures of an item from subpopulation $i$ in the interval $(a, b)$. If the number of PM actions is considered for items from heterogeneous populations, the expected cost function is given by

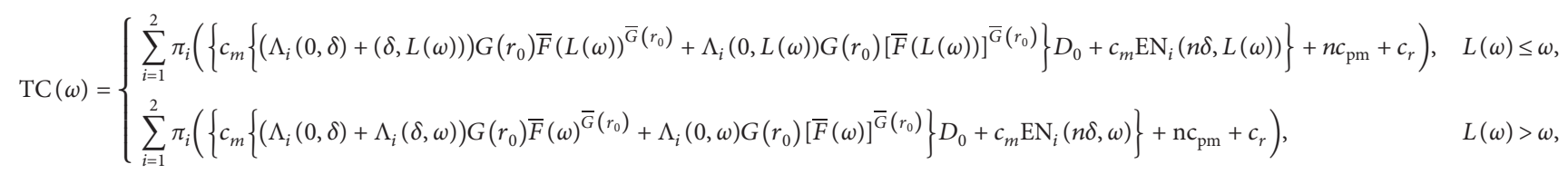

where

$$
D_{0}= \begin{cases}1, & n \geq 1 \\ 0, & n=0\end{cases}
$$

We determine the relevant set of joint probabilities for derivations as $\alpha(\gamma, \omega)$; for the probability that the item is chosen from the weak subpopulation and that the PM will be performed at $n \delta, n=0,1,2,3, \ldots, \alpha(\gamma, \omega)$ is given by

$$
\alpha(\gamma, \omega)=\pi_{1}\left\{\exp \left\{-N_{1}(0, \omega)\right\} D_{1}+\sum_{n=1}^{\infty} \int \ldots \int \prod_{j=1}^{n} \lambda_{1}\left(s_{j}\right) \exp \left\{-N_{1}(0, \omega)\right\} \mathrm{d} s_{1} \mathrm{~d} s_{2}, \ldots, \mathrm{d} s_{n}\right\},
$$

where

$$
D_{1}=\left\{\begin{array}{l}
1, \frac{\bar{F}_{2}\left(\pi_{2}\right) \pi_{2}}{\left(\prod_{j=1}^{n} \lambda_{1}\left(s_{j}\right) / \lambda_{2}\left(s_{j}\right) \bar{F}_{1}\left(\pi_{1}\right) \pi_{1}+\bar{F}_{2}\left(\pi_{2}\right) \pi_{2}\right)} \geq \gamma, \\
0, \quad \frac{\bar{F}_{2}\left(\pi_{2}\right) \pi_{2}}{\left(\prod_{j=1}^{n} \lambda_{1}\left(s_{j}\right) / \lambda_{2}\left(s_{j}\right) \bar{F}_{1}\left(\pi_{1}\right) \pi_{1}+\bar{F}_{2}\left(\pi_{2}\right) \pi_{2}\right)}<\gamma .
\end{array}\right.
$$

Similarly, for the probability that the item is chosen from the strong subpopulation and that the PM is performed at $n \delta, n=0,1,2,3, \ldots, \beta(\gamma, \omega)$ is given by

$$
\beta(\gamma, \omega)=\pi_{2}\left\{\exp \left\{-N_{2}(0, \omega)\right\} D_{2}+\sum_{n=1}^{\infty} \int \ldots \int \prod_{j=1}^{n} \lambda_{2}\left(s_{j}\right) \exp \left\{-N_{2}(0, \omega)\right\} \mathrm{d} s_{1} \mathrm{~d} s_{2}, \ldots, \mathrm{d} s_{n}\right\},
$$

where

$$
D_{2}= \begin{cases}1, & \prod_{j=1}^{n} \frac{\lambda_{1}\left(s_{j}\right)}{\lambda_{2}\left(s_{j}\right)} \frac{\bar{F}_{1}\left(\pi_{1}\right)}{\bar{F}_{2}\left(\pi_{2}\right)} \frac{\pi_{1}}{\pi_{2}} \leq \frac{1-\gamma}{\gamma}, \\ 0, & \prod_{j=1}^{n} \frac{\lambda_{1}\left(s_{j}\right)}{\lambda_{2}\left(s_{j}\right)} \frac{\bar{F}_{1}\left(\pi_{1}\right)}{\bar{F}_{2}\left(\pi_{2}\right)} \frac{\pi_{1}}{\pi_{2}}>\frac{1-\gamma}{\gamma},\end{cases}
$$


TABLE 1: Optimal set of parameters and the corresponding minimum ECR for different settings of $\left(c_{m}, c_{\mathrm{pm}}, c_{r}\right)$ to investigate the optimal length of the warranty period $\omega^{*}$ when $\pi_{1}=0.1$ and $\pi_{2}=0.9$.

\begin{tabular}{|c|c|c|c|c|c|}
\hline \multirow{2}{*}{$c_{\mathrm{pm}}$} & \multirow{2}{*}{$c_{m}$} & \multicolumn{2}{|c|}{$c_{r}=10$} & \multicolumn{2}{|c|}{$c_{r}=20$} \\
\hline & & $\omega^{*}$ & ECR & $\omega^{*}$ & ECR \\
\hline \multirow{5}{*}{1} & 1 & 1.32 & 1.4601 & 1.45 & 2.5691 \\
\hline & 2 & 1.21 & 2.9372 & 1.35 & 5.1554 \\
\hline & 3 & 1.15 & 4.4311 & 1.28 & 7.7588 \\
\hline & 4 & 1.10 & 5.9414 & 1.22 & 10.3791 \\
\hline & 5 & 1.07 & 7.4679 & 1.17 & 13.0162 \\
\hline \multirow{5}{*}{2} & 1 & 1.38 & 1.7928 & 1.46 & 2.9018 \\
\hline & 2 & 1.26 & 3.6027 & 1.37 & 5.8208 \\
\hline & 3 & 1.20 & 5.4295 & 1.32 & 8.7570 \\
\hline & 4 & 1.15 & 7.2730 & 1.25 & 11.7101 \\
\hline & 5 & 1.11 & 9.1329 & 1.21 & 14.6801 \\
\hline \multirow{5}{*}{3} & 1 & 1.39 & 2.1255 & 1.50 & 3.2344 \\
\hline & 2 & 1.31 & 4.2682 & 1.40 & 6.4862 \\
\hline & 3 & 1.25 & 6.4278 & 1.33 & 9.7551 \\
\hline & 4 & 1.19 & 8.6043 & 1.26 & 13.0411 \\
\hline & 5 & 1.14 & 10.7973 & 1.23 & 16.3439 \\
\hline \multirow{5}{*}{4} & 1 & 1.42 & 2.4582 & 1.49 & 3.5671 \\
\hline & 2 & 1.33 & 4.9336 & 1.42 & 7.1516 \\
\hline & 3 & 1.28 & 7.4261 & 1.31 & 10.7533 \\
\hline & 4 & 1.22 & 9.9354 & 1.28 & 14.3720 \\
\hline & 5 & 1.17 & 12.4615 & 1.24 & 18.0077 \\
\hline \multirow{5}{*}{5} & 1 & 1.47 & 2.79085 & 1.52 & 3.8998 \\
\hline & 2 & 1.36 & 5.5990 & 1.44 & 7.8170 \\
\hline & 3 & 1.30 & 8.4243 & 1.33 & 77.7514 \\
\hline & 4 & 1.22 & 11.2665 & 1.29 & 15.7029 \\
\hline & 5 & 1.19 & 14.1255 & 1.28 & 19.6713 \\
\hline
\end{tabular}

TABLE 2: Optimal set of parameters and the corresponding minimum ECR for different settings of $\left(c_{m}, c_{\mathrm{pm}}, c_{r}\right)$ to investigate the optimal length of warranty period $\omega^{*}$ when $\pi_{1}=0.2$ and $\pi_{2}=0.8$.

\begin{tabular}{|c|c|c|c|c|c|}
\hline \multirow{2}{*}{$c_{\mathrm{pm}}$} & \multirow{2}{*}{$c_{m}$} & \multicolumn{2}{|c|}{$c_{r}=10$} & \multicolumn{2}{|c|}{$c_{r}=20$} \\
\hline & & $\omega^{*}$ & ECR & $\omega^{*}$ & ECR \\
\hline \multirow{5}{*}{1} & 1 & 1.30 & 2.9214 & 1.45 & 5.1418 \\
\hline & 2 & 1.21 & 5.8731 & 1.34 & 10.3142 \\
\hline & 3 & 1.14 & 8.8544 & 1.25 & 15.5171 \\
\hline & 4 & 1.09 & 11.8650 & 1.21 & 20.7500 \\
\hline & 5 & 1.05 & 14.9045 & 1.18 & 26.0126 \\
\hline \multirow{5}{*}{2} & 1 & 1.37 & 3.5875 & 1.49 & 5.8078 \\
\hline & 2 & 1.26 & 7.2055 & 1.33 & 11.6465 \\
\hline & 3 & 1.17 & 10.8535 & 1.28 & 17.5156 \\
\hline & 4 & 1.14 & 14.5310 & 1.25 & 23.4148 \\
\hline & 5 & 1.11 & 18.2378 & 1.21 & 29.3440 \\
\hline \multirow{5}{*}{3} & 1 & 1.41 & 4.2536 & 1.51 & 6.4739 \\
\hline & 2 & 1.31 & 8.5379 & 1.37 & 12.9787 \\
\hline & 3 & 1.21 & 12.8523 & 1.32 & 19.5140 \\
\hline & 4 & 1.17 & 17.1965 & 1.27 & 26.0796 \\
\hline & 5 & 1.15 & 21.5702 & 1.23 & 32.6752 \\
\hline \multirow{5}{*}{4} & 1 & 1.43 & 4.9197 & 1.49 & 7.1400 \\
\hline & 2 & 1.34 & 9.8702 & 1.40 & 14.3109 \\
\hline & 3 & 1.25 & 14.8509 & 1.34 & 21.5124 \\
\hline & 4 & 1.21 & 19.8619 & 1.28 & 28.7443 \\
\hline & 5 & 1.17 & 24.9021 & 1.27 & 36.0062 \\
\hline \multirow{5}{*}{5} & 1 & 1.47 & 5.58581 & 1.52 & 7.8061 \\
\hline & 2 & 1.34 & 11.2024 & 1.42 & 15.6431 \\
\hline & 3 & 1.28 & 16.8494 & 1.35 & 23.5108 \\
\hline & 4 & 1.23 & 22.5266 & 1.30 & 31.4089 \\
\hline & 5 & 1.20 & 28.2336 & 1.27 & 39.3372 \\
\hline
\end{tabular}

TABLE 3: Optimal set of parameters and the corresponding minimum ECR for different settings of $\left(c_{m}, c_{\mathrm{pm}}, c_{r}\right)$ to investigate the optimal length of warranty period $\omega^{*}$ when $\pi_{1}=0.3$ and $\pi_{2}=0.7$.

\begin{tabular}{|c|c|c|c|c|c|}
\hline \multirow[b]{2}{*}{$c_{\mathrm{pm}}$} & \multirow[b]{2}{*}{$c_{m}$} & \multicolumn{2}{|c|}{$c_{r}=10$} & \multicolumn{2}{|c|}{$c_{r}=20$} \\
\hline & & $\omega^{*}$ & ECR & $\omega^{*}$ & ECR \\
\hline \multirow{5}{*}{1} & 1 & 1.30 & 4.3839 & 1.47 & 7.7181 \\
\hline & 2 & 1.20 & 8.8075 & 1.32 & 15.4765 \\
\hline & 3 & 1.11 & 13.2701 & 1.26 & 23.2748 \\
\hline & 4 & 1.07 & 17.7709 & 1.21 & 31.1125 \\
\hline & 5 & 1.05 & 22.3095 & 1.17 & 38.9893 \\
\hline \multirow{5}{*}{2} & 1 & 1.37 & 5.3842 & 1.47 & 8.7183 \\
\hline & 2 & 1.22 & 10.8084 & 1.38 & 17.4770 \\
\hline & 3 & 1.17 & 16.2719 & 1.28 & 26.2758 \\
\hline & 4 & 1.12 & 21.7742 & 1.24 & 35.1141 \\
\hline & 5 & 1.09 & 27.3148 & 1.20 & 43.9917 \\
\hline \multirow{5}{*}{3} & 1 & 1.44 & 6.3844 & 1.52 & 9.7185 \\
\hline & 2 & 1.26 & 12.8091 & 1.41 & 19.4775 \\
\hline & 3 & 1.21 & 19.2733 & 1.30 & 29.2767 \\
\hline & 4 & 1.17 & 25.7766 & 1.27 & 39.1155 \\
\hline & 5 & 1.14 & 32.3186 & 1.23 & 48.9938 \\
\hline \multirow{5}{*}{4} & 1 & 1.43 & 7.3847 & 1.49 & 10.7187 \\
\hline & 2 & 1.29 & 14.8097 & 1.41 & 21.4780 \\
\hline & 3 & 1.27 & 22.2744 & 1.33 & 32.2775 \\
\hline & 4 & 1.20 & 29.7786 & 1.30 & 43.1168 \\
\hline & 5 & 1.17 & 37.3217 & 1.26 & 53.9957 \\
\hline \multirow{5}{*}{5} & 1 & 1.47 & 8.38489 & 1.53 & 11.7189 \\
\hline & 2 & 1.34 & 16.8102 & 1.40 & 23.4785 \\
\hline & 3 & 1.27 & 25.2755 & 1.35 & 35.2783 \\
\hline & 4 & 1.23 & 33.7803 & 1.30 & 47.1181 \\
\hline & 5 & 1.19 & 42.3243 & 1.27 & 58.9975 \\
\hline
\end{tabular}

TABLE 4: Optimal set of parameters and the corresponding minimum ECR for different settings of $\left(c_{m}, c_{\mathrm{pm}}, c_{r}\right)$ to investigate the optimal length of warranty period $\omega^{*}$ when $\pi_{1}=0.4$ and $\pi_{2}=0.6$.

\begin{tabular}{|c|c|c|c|c|c|}
\hline \multirow{2}{*}{$c_{\mathrm{pm}}$} & \multirow[b]{2}{*}{$c_{m}$} & \multicolumn{2}{|c|}{$c_{r}=10$} & \multicolumn{2}{|c|}{$c_{r}=20$} \\
\hline & & $\omega^{*}$ & ECR & $\omega^{*}$ & ECR \\
\hline \multirow{5}{*}{1} & 1 & 1.27 & 5.8477 & 1.47 & 10.2980 \\
\hline & 2 & 1.16 & 11.7406 & 1.39 & 29.5430 \\
\hline & 3 & 1.10 & 17.6779 & 1.24 & 31.0320 \\
\hline & 4 & 1.06 & 23.6588 & 1.30 & 59.2699 \\
\hline & 5 & 1.03 & 29.6828 & 1.18 & 51.9460 \\
\hline \multirow{5}{*}{2} & 1 & 1.34 & 7.1828 & 1.44 & 11.6331 \\
\hline & 2 & 1.23 & 14.4113 & 1.34 & 23.3125 \\
\hline & 3 & 1.15 & 21.6847 & 1.27 & 35.0376 \\
\hline & 4 & 1.12 & 29.0022 & 1.23 & 46.8079 \\
\hline & 5 & 1.08 & 36.3635 & 1.19 & 58.6231 \\
\hline \multirow{5}{*}{3} & 1 & 1.42 & 8.5179 & 1.43 & 12.9682 \\
\hline & 2 & 1.26 & 17.0818 & 1.38 & 25.9827 \\
\hline & 3 & 1.19 & 25.6909 & 1.30 & 39.0431 \\
\hline & 4 & 1.15 & 34.3446 & 1.25 & 52.1489 \\
\hline & 5 & 1.12 & 43.0424 & 1.22 & 65.2997 \\
\hline \multirow{5}{*}{4} & 1 & 1.42 & 9.8530 & 1.43 & 14.3033 \\
\hline & 2 & 1.28 & 19.7522 & 1.40 & 28.6529 \\
\hline & 3 & 1.24 & 29.6967 & 1.34 & 43.0485 \\
\hline & 4 & 1.20 & 39.6862 & 1.28 & 57.4897 \\
\hline & 5 & 1.15 & 49.7203 & 1.24 & 71.9761 \\
\hline \multirow{5}{*}{5} & 1 & 1.41 & 11.1881 & 1.51 & 15.6383 \\
\hline & 2 & 1.34 & 22.4224 & 1.41 & 31.3231 \\
\hline & 3 & 1.27 & 33.7024 & 1.33 & 47.0540 \\
\hline & 4 & 1.23 & 45.0275 & 1.30 & 62.8304 \\
\hline & 5 & 1.20 & 56.3974 & 1.27 & 78.6552 \\
\hline
\end{tabular}


TABLE 5: Optimal set of parameters and the corresponding minimum ECR for different settings of repair time limit and shape parameter for power law process to investigate the optimal length of warranty period $\omega^{*}$ when $\pi_{1}=0.1$ and $\pi_{2}=0.9$.

\begin{tabular}{|c|c|c|c|c|c|c|}
\hline \multirow{2}{*}{$r_{0}$} & \multicolumn{2}{|c|}{$\kappa_{1}=2.0$} & \multicolumn{2}{|c|}{$\kappa_{1}=2.5$} & \multicolumn{2}{|c|}{$\kappa_{1}=3.0$} \\
\hline & $\omega^{*}$ & ECR & $\omega^{*}$ & ECR & $\omega^{*}$ & ECR \\
\hline $1 / 12$ & 1.20 & 2.9391 & 1.21 & 2.9389 & 1.21 & 2.9372 \\
\hline $2 / 12$ & 1.07 & 2.9871 & 1.06 & 2.9869 & 1.05 & 2.9852 \\
\hline $3 / 12$ & 1.00 & 3.0301 & 0.97 & 3.0296 & 0.95 & 3.0275 \\
\hline $4 / 12$ & 0.95 & 3.0688 & 0.92 & 3.0679 & 0.89 & 3.0650 \\
\hline $5 / 12$ & 0.91 & 3.1038 & 0.88 & 3.1021 & 0.85 & 3.0984 \\
\hline $6 / 12$ & 0.88 & 3.1354 & 0.85 & 3.1330 & 0.82 & 3.1282 \\
\hline $7 / 12$ & 0.85 & 3.1642 & 0.82 & 3.1609 & 0.79 & 3.1550 \\
\hline $8 / 12$ & 0.83 & 3.1903 & 0.80 & 3.1861 & 0.77 & 3.1792 \\
\hline $9 / 12$ & 0.82 & 3.2141 & 0.78 & 3.2090 & 0.76 & 3.2011 \\
\hline $10 / 12$ & 0.80 & 3.2357 & 0.77 & 3.2297 & 0.74 & 3.2208 \\
\hline $11 / 12$ & 0.79 & 3.2555 & 0.76 & 3.2486 & 0.73 & 3.2388 \\
\hline $12 / 12$ & 0.78 & 3.2735 & 0.75 & 3.2658 & 0.72 & 3.2551 \\
\hline
\end{tabular}

TABLE 6: Optimal set of parameters and the corresponding minimum ECR for different settings of repair time limit and shape parameter for the power law process to investigate the optimal length of warranty period $\omega^{*}$ when $\kappa_{1}=3, \lambda_{1}=2$, and $\kappa_{2}=1.5, \lambda_{2}=1$

\begin{tabular}{lcccccc}
\hline & \multicolumn{2}{c}{$\pi_{1}=0.2, \pi_{2}=$} & \multicolumn{2}{c}{$\pi_{1}=0.3, \pi_{2}=$} & \multicolumn{2}{c}{$\pi_{1}=0.4, \pi_{2}=$} \\
$r_{0}$ & \multicolumn{2}{c}{0.8} & \multicolumn{2}{c}{0.7} & \multicolumn{2}{c}{0.6} \\
& $\omega^{*}$ & ECR & $\omega^{*}$ & ECR & $\omega^{*}$ & ECR \\
\hline $1 / 12$ & 1.21 & 5.8731 & 1.20 & 8.8075 & 1.16 & 11.7406 \\
$2 / 12$ & 1.03 & 5.9619 & 1.00 & 8.9301 & 0.97 & 11.8894 \\
$3 / 12$ & 0.93 & 6.0399 & 0.91 & 9.0370 & 0.88 & 12.0180 \\
$4 / 12$ & 0.87 & 6.1087 & 0.85 & 9.1307 & 0.83 & 12.1300 \\
$5 / 12$ & 0.83 & 6.1698 & 0.81 & 9.2136 & 0.79 & 12.2286 \\
$6 / 12$ & 0.80 & 6.2243 & 0.78 & 9.2873 & 0.76 & 12.3160 \\
$7 / 12$ & 0.78 & 6.2731 & 0.76 & 9.3533 & 0.73 & 12.3939 \\
$8 / 12$ & 0.76 & 6.3171 & 0.74 & 9.4124 & 0.71 & 12.4636 \\
$9 / 12$ & 0.74 & 6.3567 & 0.72 & 9.4657 & 0.70 & 12.5262 \\
$10 / 12$ & 0.73 & 6.3926 & 0.71 & 9.5139 & 0.69 & 12.5828 \\
$11 / 12$ & 0.71 & 6.4251 & 0.70 & 9.5574 & 0.67 & 12.6339 \\
$12 / 12$ & 0.69 & 6.4546 & 0.68 & 9.6134 & 0.67 & 12.6801 \\
\hline
\end{tabular}

which obviously correspond to

$$
\alpha(\gamma, \omega)+\beta(\gamma, \omega)=1
$$

TABLE 7: Optimal set of parameters and the corresponding minimum ECR for different settings of repair time limit to investigate the optimal length of warranty period $\omega^{*}$ when $\kappa_{1}=17.1668, \lambda_{1}=$ 0.0815 and $\kappa_{2}=2.5289, \lambda_{1}=0.2513$

\begin{tabular}{lcccccc}
\hline & \multicolumn{2}{c}{$\pi_{1}=0.2, \pi_{2}=$} & \multicolumn{2}{c}{$\pi_{1}=0.3, \pi_{2}=$} & \multicolumn{2}{c}{$\pi_{1}=0.4, \pi_{2}=$} \\
$r_{0}$ & \multicolumn{2}{c}{0.8} & \multicolumn{2}{c}{0.7} & \multicolumn{2}{c}{0.6} \\
& $\omega^{*}$ & ECR & $\omega^{*}$ & ECR & $\omega^{*}$ & ECR \\
\hline $1 / 12$ & 0.489 & 27.1398 & 0.489 & 43.9342 & 0.495 & 63.6260 \\
$2 / 12$ & 0.462 & 27.4556 & 0.465 & 44.4177 & 0.468 & 64.2857 \\
$3 / 12$ & 0.447 & 27.7375 & 0.450 & 44.8505 & 0.453 & 64.8777 \\
$4 / 12$ & 0.438 & 27.9917 & 0.441 & 45.2415 & 0.444 & 65.4133 \\
$5 / 12$ & 0.429 & 28.2222 & 0.432 & 45.5964 & 0.435 & 65.9004 \\
$6 / 12$ & 0.423 & 28.4318 & 0.426 & 45.9195 & 0.432 & 66.3442 \\
$7 / 12$ & 0.420 & 28.6229 & 0.423 & 46.2142 & 0.426 & 66.7493 \\
$8 / 12$ & 0.414 & 28.7973 & 0.417 & 46.4835 & 0.423 & 67.1199 \\
$9 / 12$ & 0.411 & 28.9568 & 0.414 & 46.7298 & 0.417 & 67.4590 \\
$10 / 12$ & 0.408 & 29.1027 & 0.411 & 46.9552 & 0.414 & 67.7695 \\
$11 / 12$ & 0.405 & 29.2363 & 0.408 & 47.1618 & 0.414 & 68.0541 \\
$12 / 12$ & 0.402 & 29.3588 & 0.408 & 47.3511 & 0.411 & 68.3149 \\
\hline
\end{tabular}

The expected cost rate can be obtained by dividing the expected cost by the expected duration from equation (3). When $L(\omega) \leq \omega$, the expected cost rate can be obtained by

$$
\operatorname{ECR}(\omega)=\frac{\sum_{i=1}^{2} \pi_{i}\left(\left\{c_{m}\left\{\left(\Lambda_{i}(0, \delta)+(\delta, L(\omega))\right) G\left(r_{0}\right) \bar{F}(L(\omega))^{\bar{G}\left(r_{0}\right)}+\Lambda_{i}(0, L(\omega)) G\left(r_{0}\right)[\bar{F}(L(\omega))]^{\left.\bar{G}\left(r_{0}\right)\right\}} D_{0}+c_{m} \mathrm{EN}_{i}(n \delta, L(\omega))\right\}+\mathrm{nc} c_{\mathrm{pm}}+c_{r}\right)\right.}{\int_{0}^{n \cdot \delta}\left(\pi_{1} \bar{F}_{1}(t)+\pi_{2} \bar{F}_{2}(t)\right) \mathrm{d} t}
$$

where

$$
N_{i}(n \delta, \omega)=\Lambda_{i}(n \delta, \omega) \cdot G\left(r_{0}\right) \cdot\left[\bar{F}_{i}(\omega)\right]^{\bar{G}\left(r_{0}\right)}, \quad i=1,2 .
$$

When $L(\omega)>\omega$, the expected cost rate can be obtained 


$$
\operatorname{ECR}(\omega)=\frac{\sum_{i=1}^{2} \pi_{i}\left(\left\{c_{m}\left\{\left(\Lambda_{i}(0, \delta)+\Lambda_{i}(\delta, \omega)\right) G\left(r_{0}\right) \bar{F}(\omega)^{\bar{G}\left(r_{0}\right)}+\Lambda_{i}(0, \omega) G\left(r_{0}\right)[\bar{F}(\omega)]^{\bar{G}\left(r_{0}\right)}\right\} D_{0}+c_{m} \mathrm{EN}_{i}(n \delta, \omega)\right\}+\mathrm{nc} c_{\mathrm{pm}}+c_{r}\right)}{\int_{0}^{n \cdot \delta}\left(\pi_{1} \bar{F}_{1}(t)+\pi_{2} \bar{F}_{2}(t)\right) \mathrm{d} t},
$$

where

$$
N_{i}(n \delta, \omega)=\Lambda_{i}(n \delta, \omega) \cdot G\left(r_{0}\right) \cdot\left[\bar{F}_{i}(\omega)\right]^{\bar{G}\left(r_{0}\right)}, \quad i=1,2 .
$$

3.2. Optimization. By minimizing $\operatorname{ECR}(\omega)$ formulated in equation (37), the optimal length of the warranty period for a repairable product under the information-based repair-replacement policy can be derived, and the corresponding minimum ECR is readily evaluated. The optimal length of the warranty period, denoted by $\omega^{*}$, is determined as

$$
\operatorname{ECR}\left(\omega^{*}\right)=\min _{\omega} \operatorname{ECR}(\omega)
$$

To find the optimal solution with respect to $\omega$ for equation (37), we utilize the Nelder-Mead downhill simplex method [31], which does not require the calculation of the derivatives and is most widely used to solve such a nonlinear equation.

\section{Numerical Analysis}

In this section, numerical examples which illustrate the application of the proposed approach will be given. Due to the complex nonlinear functional forms of equations (34) and (36), we use the Nelder-Mead downhill simplex method [31] to obtain an optimal strategy for the given optimization problem to minimize the ECR.

For the purpose of numerical calculations for this example, we consider various choices of cost parameters to find the optimal length of the warranty period and to study the pattern changes of the optimal warranty length and its expected cost rate. Throughout this example, we use a year as the unit of time. As for the failure time, we can use the power law process with the intensity functions $f_{1}(t)$ for items from the weak population and $f_{2}(t)$ for items from the strong population which are given by

$$
f_{1}(t)=\frac{\kappa_{1}}{\lambda_{1}}\left(\frac{t}{\lambda_{1}}\right)^{\kappa_{1}-1}, f_{2}(t)=\frac{\kappa_{2}}{\lambda_{2}}\left(\frac{t}{\lambda_{2}}\right)^{\kappa_{2}-1}, \quad t \geq 0, \kappa_{1}, \lambda_{1}, \kappa_{2}, \lambda_{2}>0
$$

The repair time is considered to follow two-parameter Weibull distribution in which its $p d f$ is given by

$$
g(y)=\frac{\nu}{\eta}\left(\frac{y}{\eta}\right)^{\nu-1} e^{-(y / \eta)^{\nu}}, \quad y \geq 0, \nu, \eta>0 .
$$

Tables 1-4 show the optimal length of the warranty period, denoted by $\omega^{*}$ and its resulting values of $\operatorname{EC~}\left(\omega^{*}\right)$ for different combinations of $c_{m}, c_{\mathrm{pm}}$, and $c_{r}$. Here, we assume that $r_{0}=1 / 12$, where $r_{0}$ is the repair time limit set by the manufacturer. When $\kappa_{1}=3, \lambda_{1}=2, \kappa_{2}=1.5, \lambda_{2}=1$ and $\pi_{1}$ and $\pi_{2}$ are given by 0.1 and 0.9 , respectively, in Table $1, \nu=$ $2, \eta=1$ are given for repair time. For instance, in Table 1, when the replacement cost is equal to 10 and $c_{m}=1, c_{\mathrm{pm}}=1$, $\omega^{*}$ equals 1.32. Its expected cost rate is 1.4601. Similarly, when the replacement cost is equal to 20 and $c_{m}=1, c_{\mathrm{pm}}=1$, $\omega^{*}$ equals 1.45. Its expected cost rate is 2.5691. As a minimal repair cost, a replacement cost, and a PM cost increase, then the ECR increases. As a minimal repair cost increases, the optimal length of the warranty period decreases. On the other hand, as PM cost and replacement cost increase, the optimal length of the warranty period increases. For Table 2, $\pi_{1}$ and $\pi_{2}$ are given by 0.2 and 0.8 , respectively, and other parameters are the same as the case in Table 1. In Table 2, when the replacement cost equals 10 and $c_{m}=1, c_{\mathrm{pm}}=1, \omega^{*}$ equals 1.30. Its expected cost rate is 2.9214. When the replacement cost equals 20 and $c_{m}=1, c_{\mathrm{pm}}=1, \omega^{*}$ equals 1.45 . Its expected cost rate is 5.1418 . For Table $3, \pi_{1}$ and $\pi_{2}$ are given by 0.3 and 0.7 , respectively, and other parameters are the same as the case in Table 2 . For Table $4, \pi_{1}$ and $\pi_{2}$ are given by 0.4 and 0.6 , respectively, and other parameters are the same as the case in Table 3.

In Table 5, when the replacement cost is equal to 10 and $c_{m}=2, c_{\mathrm{pm}}=1, \kappa_{1}=2$ for power law process for failure time, when $\pi_{1}=0.1$ and $\pi_{2}=0.9$, and when the repair time limit equals $1 / 12$, then $\omega^{*}$ equals 1.20 . Its expected cost rate is 2.9391. Similarly, when $\kappa_{1}=2.5$ for the power law process for failure time, $\omega^{*}$ equals 1.21. Its expected cost rate is 2.9389. As the shape parameter $\kappa_{1}$ increases, then the ECR and the optimal length of the warranty period decrease. In Table 6, when the replacement cost is equal to 10 and $c_{m}=$ $2, c_{\mathrm{pm}}=1, \kappa_{1}=3$ for power law process for failure time, when $\pi_{1}=0.2$ and $\pi_{2}=0.8$, and when the repair time limit equals $1 / 12$, then $\omega^{*}$ equals 1.21 . Its expected cost rate is 5.8731. Similarly, when $\pi_{1}=0.3$ and $\pi_{2}=0.7$, $\omega^{*}$ equals 1.20. Its expected cost rate is 8.8075 .

For the real application, we are going to show a practical example using the field data from Yang et al. [32]. In Yang et al.'s study [32], the authors considered the reliability assessment of smart electricity meter (SEM) tested in a multiple stresses accelerating environment. They conducted the accelerated degradation test on SEM with respect to the billing function. Temperature, relative humidity, and electricity current were selected as the accelerating stresses and the combination of stresses can be used for the accelerated degradation test. It was assumed that Weibull distribution could be used to describe the pseudo lifetime data of SEM. In Lin et al.'s study [33], the likelihood ratio test is used to test the null hypothesis of the homogeneous population against 
the alternative hypothesis of heterogeneous populations using the field data from Yang et al. [32]. The normal operational temperature and electricity current for SEM range from $-40^{\circ} \mathrm{C}$ to $60^{\circ} \mathrm{C}$ and $0 \mathrm{~A}$ to $10 \mathrm{~A}$, respectively [32]. When the temperature is $80^{\circ} \mathrm{C}$, the relative humidity is $80 \%$, the electricity current is $20 \mathrm{~A}$, and the sample size is 64 , the EM algorithm is used to estimate the parameters of mixture Weibull distribution, given by $\kappa_{1}=17.1668, \lambda_{1}=0.0815$ and $\kappa_{2}=2.5289, \lambda_{1}=0.2513$ [33]. In order to build a realistic degradation model of SEM, we simulate the real operation conditions of SEM. Table 7 presents the optimal warranty length and its ECR for different setting and conditions. In Table 7, when the replacement cost is equal to 10 and $c_{m}=$ $2, c_{\mathrm{pm}}=1, \pi_{1}=0.2, \pi_{2}=0.8$, and the repair time limit equals $1 / 12$, then $\omega^{*}$ equals 0.489 . Its expected cost rate is 27.1398 .

\section{Concluding Remarks}

We extend the situation where minimal repair and replacement can be considered simultaneously when product failures occur during the warranty period. Under the information-based repair-replacement policy studied in this paper, the failed item is either minimally repaired or replaced depending on whether the repair time exceeds a prespecified threshold or not. If the product reaches the preventive age without failures, the product is replaced preventively. For customers, an information-based repairreplacement policy could be more desirable than an unconditional replacement of the failed product, since the customer has other choices, especially for a high-priced product.

In this paper, we formulate the information-based repair-replacement policy model and develop the formula to evaluate the expected cost rate during the product life cycle. Then, by applying the Nelder-Mead downhill simplex method, we find the optimal length of the warranty period and its corresponding expected cost rate. The effects of parameters of the intensity function for the failure times on the optimal length of the warranty period are studied numerically. In addition, the impact of the repair time threshold on the optimal length of the warranty period is investigated. Research regarding the information-based repair-replacement policy incorporating the several types of warranty policy would be helpful in the future.

\section{Nomenclature}

NHPP:

PM, CM:

FR, PR:

ECR:

FRRW:

r.v.:

$$
\begin{aligned}
& T, Y: \\
& r_{0}: \\
& \delta: \\
& \omega: \\
& c_{m}, c_{r}, c_{\mathrm{pm}}: \\
& \\
& \pi_{1}, \pi_{2}: \\
& \lambda(\cdot): \\
& \lambda_{i}^{p}(t):
\end{aligned}
$$

$r . v$. 's representing failure time and repair time of the product, respectively

Repair time threshold PM cycle Length of the warranty period Unit minimal repair cost, unit replacement cost, and unit $\mathrm{PM}$ cost, respectively

Proportions of the weak items and the strong items, respectively Failure rate function for an item Failure rate function after a PM action for an item from subpopulation $i$ ( $i=1$, weak population, and $i=2$, strong population)

$f(\cdot), F(\cdot), \bar{F}(\cdot)$ :

$p d f, c d f$, survival function of failure time $T$, respectively

$F_{1}(\cdot), f_{1}(\cdot), \bar{F}_{1}(\cdot), \lambda_{1}(\cdot)$ : $p d f, c d f$, survival function and failure rate, respectively, for an item from a weak subpopulation

$F_{2}(\cdot), f_{2}(\cdot), \bar{F}_{2}(\cdot), \lambda_{2}(\cdot)$ : pdf, $c d f$, survival function and failure rate, respectively, for an item from a strong subpopulation $g(\cdot), G(\cdot), \bar{G}(\cdot)$ : $p d f, c d f$, survival function of repair time $Y$, respectively

$L(\cdot):$

$q(\cdot), Q(\cdot), \bar{Q}(\cdot):$

$N(a, b):$

$\mathrm{TC}(\omega)$ :

$\operatorname{EC}(\omega):$

$\operatorname{ECR}(\omega)$ Elapsed time to the first replacement of the item from a heterogeneous population $p d f$, $c d f$, survival function of $L(\cdot)$, respectively

Number of failures of an item in the interval $(a, b)$

Total cost incurred during the warranty period Expected total cost incurred during the warranty period Long-run expected cost rate during the warranty period.

\section{Data Availability}

The simulation data used to support the findings of this study are available from the corresponding author upon request.

\section{Conflicts of Interest}

The author declares that there are no conflicts of interest regarding the publication of this paper.

\section{Acknowledgments}

This work was supported by the National Research Foundation of Korea (NRF) grant funded by the Korea government (MSIT) (NRF-2020R1F1A104823711). 


\section{References}

[1] Y. Wang, Z. Liu, and Y. Liu, "Optimal preventive maintenance strategy for repairable items under two-dimensional warranty," Reliability Engineering \& System Safety, vol. 142, pp. 326-333, 2015.

[2] Y.-S. Huang, C.-D. Huang, and J.-W. Ho, "A customized twodimensional extended warranty with preventive maintenance," European Journal of Operational Research, vol. 257, no. 3, pp. 971-978, 2017.

[3] S. Bouguerra, A. Chelbi, and N. Rezg, "A decision model for adopting an extended warranty under different maintenance policies," International Journal of Production Economics, vol. 135, no. 2, pp. 840-849, 2012.

[4] C. Su and X. Wang, "A two-stage preventive maintenance optimization model incorporating two-dimensional extended warranty," Reliability Engineering \& System Safety, vol. 155, pp. 169-178, 2016.

[5] X. Wang and C. Su, "A two-dimensional preventive maintenance strategy for items sold with warranty," International Journal of Production Research, vol. 54, no. 19, pp. 5901-5915, 2016.

[6] J. Wang, Z. Zhou, and H. Peng, "Flexible decision models for a two-dimensional warranty policy with periodic preventive maintenance," Reliability Engineering \& System Safety, vol. 162, pp. 14-27, 2017.

[7] Y.-S. Huang, W.-Y. Gau, and J.-W. Ho, "Cost analysis of twodimensional warranty for products with periodic preventive maintenance," Reliability Engineering \& System Safety, vol. 134, pp. 51-58, 2015.

[8] S. N. Mujahid and M. A. Rahim, "Optimal preventive maintenance warranty policy for repairable products with periodically increasing failure rate," International Journal of Operational Research, vol. 9, no. 2, pp. 227-240, 2010.

[9] M. Park and H. Pham, "Warranty cost analysis for k-out-of-n systems with 2-D warranty," IEEE Transactions on Systems, Man, and Cybernetics-Part A: Systems and Humans, vol. 42, no. 4, pp. 947-957, 2012.

[10] M. Park, K. Mun Jung, and D. H. Park, "Optimal postwarranty maintenance policy with repair time threshold for minimal repair," Reliability Engineering \& System Safety, vol. 111, pp. 147-153, 2013.

[11] Y.-H. Chien, "The optimal preventive-maintenance policy for a nhpbp repairable system under free-repair warranty," Reliability Engineering \& System Safety, vol. 188, 2019.

[12] K. M. Jung, M. Park, and D. H. Park, "System maintenance cost dependent on life cycle under renewing warranty policy," Reliability Engineering \& System Safety, vol. 95, no. 7, pp. 816-821, 2010.

[13] K. M. Jung, M. Park, and D. H. Park, "Optimal maintenance strategy for non-renewing replacement-repair warranty," Applied Stochastic Models in Business and Industry, vol. 28, no. 6, pp. 607-614, 2012.

[14] K. M. Jung, M. Park, and D. H. Park, "Cost optimization model following extended renewing two-phase warranty," Computers \& Industrial Engineering, vol. 79, pp. 188-194, 2015.

[15] C. S. Kim, I. Djamaludin, and D. N. P. Murthy, "Warranty and discrete preventive maintenance," Reliability Engineering \& System Safety, vol. 84, no. 3, pp. 301-309, 2004.

[16] M. Park, K. M. Jung, and D. H. Park, "Optimal warranty policies considering repair service and replacement service under the manufacturer's perspective," Annals of Operations Research, vol. 244, no. 1, pp. 117-132, 2016.
[17] M. Park, K. M. Jung, and D. H. Park, "A generalized age replacement policy for systems under renewing repair-replacement warranty," IEEE Transactions on Reliability, vol. 65, no. 2, pp. 604-612, 2015.

[18] M. Park, K. M. Jung, and D. H. Park, "Optimal maintenance strategy under renewable warranty with repair time threshold," Applied Mathematical Modelling, vol. 43, pp. 498-508, 2017.

[19] M. Park, K. M. Jung, and D. H. Park, "Optimization of periodic preventive maintenance policy following the expiration of two-dimensional warranty," Reliability Engineering \& System Safety, vol. 170, pp. 1-9, 2018.

[20] M. S. Finkelstein, "Minimal repair in heterogeneous populations," Journal of Applied Probability, vol. 41, no. 1, pp. 281-286, 2004.

[21] J. H. Cha, "Optimal replacement of heterogeneous items with minimal repairs," IEEE Transactions on Reliability, vol. 65, no. 2, pp. 593-603, 2016.

[22] J. H. Cha and M. Finkelstein, "Stochastic analysis of preventive maintenance in heterogeneous populations," Operations Research Letters, vol. 40, no. 5, pp. 416-421, 2012.

[23] H. Lee, J. H. Cha, and M. Finkelstein, "On information-based warranty policy for repairable products from heterogeneous population," European Journal of Operational Research, vol. 253, no. 1, pp. 204-215, 2016.

[24] J. H. Cha and M. Finkelstein, "Stochastic intensity for minimal repairs in heterogeneous populations," Journal of Applied Probability, vol. 48, no. 3, pp. 868-876, 2011.

[25] L. Yang, Z.-s. Ye, C.-G. Lee, S.-f. Yang, and R. Peng, “A twophase preventive maintenance policy considering imperfect repair and postponed replacement," European Journal of Operational Research, vol. 274, no. 3, pp. 966-977, 2019.

[26] R. Peng, B. Liu, Q. Zhai, and W. Wang, "Optimal maintenance strategy for systems with two failure modes," Reliability Engineering \& System Safety, vol. 188, pp. 624-632, 2019.

[27] M. Finkelstein, "On statistical and information-based virtual age of degrading systems," Reliability Engineering \& System Safety, vol. 92, no. 5, pp. 676-681, 2007.

[28] B. Natvig, "On information-based minimal repair and the reduction in remaining system lifetime due to the failure of a specific module," Journal of Applied Probability, vol. 27, no. 2, pp. 365-375, 1990.

[29] P. J. Boland and E. El-Neweihi, "Statistical and information based (physical) minimal repair for $\mathrm{k}$ out of $\mathrm{n}$ systems," Journal of Applied Probability, vol. 35, no. 3, pp. 731-740, 1998.

[30] M. Park, K. M. Jung, and D. H. Park, "Warranty cost analysis for second-hand products under a two-stage repair-or-full refund policy," Reliability Engineering \& System Safety, vol. 193, p. 106596, 2020.

[31] R. Rardin, Optimization in Operations Research, PrenticeHall, Upper Saddle River, NJ, USA, 1998.

[32] Z. Yang, Y. X. Chen, Y. F. Li, E. Zio, and R. Kang, "Smart electricity meter reliability prediction based on accelerated degradation testing and modeling," International Journal of Electrical Power \& Energy Systems, vol. 56, pp. 209-219, 2014.

[33] K. Lin, Y. Chen, and D. Xu, "Reliability assessment model considering heterogeneous population in a multiple stresses accelerated test," Reliability Engineering \& System Safety, vol. 165, pp. 134-143, 2017. 